\title{
Genomic Imprinting at the Porcine DIRAS3 Locus
}

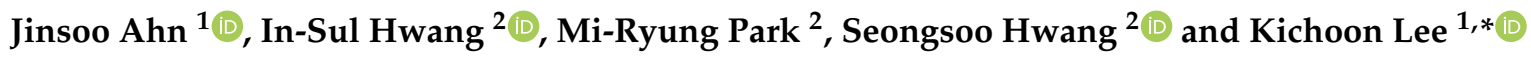 \\ 1 Functional Genomics Laboratory, Department of Animal Sciences, The Ohio State University, \\ Columbus, OH 43210, USA; ahn.134@osu.edu \\ 2 Animal Biotechnology Division, National Institute of Animal Science, Rural Development Administration, \\ Wanju 55365, Korea; insuri2642@korea.kr (I.-S.H.); mrpark45@korea.kr (M.-R.P.); hwangss@korea.kr (S.H.) \\ * Correspondence: lee.2626@osu.edu; Tel.: +1-614-688-7963
}

check for

updates

Citation: Ahn, J.; Hwang, I.-S.; Park, M.-R.; Hwang, S.; Lee, K. Genomic Imprinting at the Porcine DIRAS3 Locus. Animals 2021, 11, 1315. https://doi.org/10.3390/ani11051315

Academic Editor: Sunday Peters

Received: 25 April 2021

Accepted: 1 May 2021

Published: 3 May 2021

Publisher's Note: MDPI stays neutral with regard to jurisdictional claims in published maps and institutional affiliations.

Copyright: (c) 2021 by the authors. Licensee MDPI, Basel, Switzerland. This article is an open access article distributed under the terms and conditions of the Creative Commons Attribution (CC BY) license (https:// creativecommons.org/licenses/by/ $4.0 /)$.
Simple Summary: DNA methylation associated with one of the two alleles from parents is an important mechanism that causes a silencing of that allele, leading to expression of another allele only. There has been a lack of detailed studies on DNA methylation and expression patterns that are related to the DIRAS3 gene in pigs. The objective of this study was to provide a comprehensive overview of DNA methylation and expression associated with the DIRAS3 gene in pigs by generating an embryonic pig model and analyzing next-generation sequencing using pig embryos and adult pigs. Our results clearly showed the presence of DNA methylation near the DIRAS3 gene in pigs and high expression of DIRAS3 in the hypothalamus from adult pigs and expression of only one allele in all the tested tissues including the hypothalamus. In summary, our findings suggested DNA methylation might be related to those unique gene expression patterns during the development of pigs.

Abstract: The epigenetic mechanisms underlying genomic imprinting include DNA methylation and monoallelic expression of genes in close proximity. Although genes imprinted in humans and mice have been widely characterized, there is a lack of detailed and comprehensive studies in livestock species including pigs. The purpose of this study was to investigate a detailed methylation status and parent-of-origin-specific gene expression within the genomic region containing an underexamined porcine DIRAS3 locus. Through whole-genome bisulfite sequencing (WGBS) and RNA sequencing (RNA-seq) of porcine parthenogenetic embryos and analyses of public RNA-seq data from adult pigs, DNA methylation and monoallelic expression pattern were investigated. As a result, maternal hypermethylation at the DIRAS3 locus and hypothalamus-specific and monoallelic expression of the DIRAS3 gene were found in pigs. In conclusion, the findings from this study suggest that the presence of maternal hypermethylation, or imprints, might be maintained and related to monoallelic expression of DIRAS3 during pig development.

Keywords: DNA methylation; monoallelic expression; pigs; DIRAS3; whole-genome bisulfite sequencing; RNA-seq; hypothalamus

\section{Introduction}

Genomic imprinting plays a crucial role in mammalian development and growth [1] The epigenetic mechanisms underlying genomic imprinting include DNA methylation during mammalian embryonic development [2,3]. Differentially established DNA methylation in two parental germlines consists of epigenetic imprints and causes monoallelic expression of genes in close proximity [4]. For example, a differentially methylated region (DMR) at the SGCE / PEG10 locus serves as the imprinting control region (ICR) which forces paternal allele-specific expression in humans [5,6], mice [7,8], pigs [9], and sheep [10]. Although genes imprinted in humans and mice have been widely characterized, there is a lack of detailed and comprehensive studies in livestock species including pigs. Our recent approach of the use of whole-genome bisulfite sequencing (WGBS) and RNA sequencing 
(RNA-seq) of porcine embryos that underwent parthenogenesis has facilitated verification of the imprinting status of known imprinted clusters and identification of novel imprinted genes $[9,11]$. This facilitation was attributed to a direct sequencing comparison between parthenogenetic and biparental porcine embryos. As a result, a detailed methylation status and parent-of-origin-specific gene expression within genomic regions containing underexamined target loci, such as the DIRAS3 locus, could be further investigated.

The distinct subgroup of the Ras family member 3 (DIRAS3) gene, also known as ARHI and NOEY2, encodes a small GTPase, which belongs to the Ras superfamily and serves as a tumor suppressor against various cancers including breast and ovarian cancers [11,12]. A maternal imprint and paternal monoallelic expression of the human DIRAS3 gene have been reported to establish a functional allele that acts as a negative regulator of growth and development $[13,14]$. In pigs, although paternal expression of the DIRAS3 gene at the fetal stage [15] and in adult skeletal muscles in which expression of DIRAS3 is very low [16] was reported, DNA methylation imprints at the locus has not been investigated. In addition, according to the genotype-tissue expression (GTEx) project (https:/ / gtexportal. org/home/gene/DIRAS3, accessed 15 January 2021), in an adult human, the DIRAS3 gene was expressed the most in the hypothalamus among 54 sampled normal tissues and expressed in high levels in the nucleus accumbens (basal ganglia), ovary, and pituitary, whereas expressions in the other 50 normal human tissues were very low. However, detailed information about tissue-specific and/or monoallelic expression of the DIRAS3 gene in adult pigs is still missing. Therefore, we explored DNA methylation using our parthenogenetic model and the expression of DIRAS3 in the adult pig hypothalamus, as well as other major tissues (adipose, liver, lung, skeletal muscle, and spleen) by processing raw RNA-seq data from this study and from gene expression omnibus (GEO) series.

Here, we aimed to determine concurrence of paternal allele expression and a maternal DMR within the promoter, exon, and intron regions of the porcine DIRAS3 gene using RNA-seq and WGBS of parthenogenetic and biparental control embryos. In addition, tissuespecific and monoallelic expression of the DIRAS3 gene in adult pigs was investigated by analyzing RNA-seq data. Our comprehensive analysis of DNA methylation and imprinted gene expression status of the porcine DIRAS3 gene provides advanced groundwork for future studies of the DIRAS3 locus.

\section{Materials and Methods}

\subsection{Sample Collection and Data Used}

Animal procedures were approved by the IACUC of the National Institute of Animal Science of Korea (approval number NIAS2015-670). Porcine oocytes were collected from 50 normal Landrace $\times$ Yorkshire $\times$ Duroc $($ LYD) pigs and matured in vitro and then parthenogenetic embryos were generated by electrical stimulation described in our previous reports [9,17]. After placing into oviducts of surrogate gilts, parthenogenetic embryos were developed until day 21. Furthermore, fertilized control porcine embryos were collected on day 21 after natural mating as described previously $[9,17]$. A publicly available dataset under accession number GSE158430 in the GEO database (https: / / www.ncbi.nlm.nih.gov/geo/) was accessed on 29 November 2020. Then, raw RNA-seq data for adipose tissue, hypothalamus, liver, lungs, skeletal muscles, and spleen of two 6-month-old adult male pigs (P348 and P350) (SubSeries GSE158412), raw H3K4me1 ChIP-seq data of the P348 hypothalamus (SubSeries GSE158427), and raw CTCF ChIP-seq data of the P350 hypothalamus (SubSeries GSE158416) were downloaded. We named P348-Pig 1, p350-Pig 2.

\subsection{Whole-Genome Bisulfite Sequencing (WGBS) and RNA Sequencing (RNA-seq)}

For WGBS data generation, genomic DNA was isolated from the whole randomly collected control $(\mathrm{CN}, \mathrm{n}=3)$ and parthenote (PA, $n=3)$ embryos and processed as previously reported [9,17]. Briefly, an Accel-NGS Methyl-Seq DNA Library Kit (Swift Biosciences, Inc.; Ann Arbor, MI, USA) was used to optimize bisulfite conversion of genomic DNA according 
to the manufacturer's instructions. PCR was conducted with adapter primers and Diastar ${ }^{\mathrm{TM}}$ EF-Taq DNA polymerase (Solgent, Daejeon, Korea) under the following thermal conditions: 3 min at $95^{\circ} \mathrm{C}$ followed by 35 cycles of $30 \mathrm{~s}$ at $95^{\circ} \mathrm{C}, 30 \mathrm{~s}$ at $60{ }^{\circ} \mathrm{C}$, and $30 \mathrm{~s}$ at $72{ }^{\circ} \mathrm{C}$, and a final extension for $5 \mathrm{~min}$ at $72{ }^{\circ} \mathrm{C}$. After a bead-based clean-up, the PCR products were sequenced using a HiSeqX sequencer operated by Macrogen Inc. (Seoul, Korea) and 151 cycles for Read 1 and Read 2 were completed. The raw reads were checked for quality using FastQC (v0.11.7) (Babraham Bioinformatics, Cambridge, UK) and then trimmed and filtered out to remove adapters and reads shorter than 20 bp by using Trim Galore (v0.4.5) (Babraham Bioinformatics), leaving 846.5 (CN1), 862.1 (CN2), 866.5 (CN3), 839.7 (PA1), 856.9 (PA2), and 849.2 (PA3) million cleaned reads. Mapping to the pig reference genome (the Sscrofa11.1 assembly downloaded from https: / / www.ncbi.nlm.nih.gov/assembly/GCF_000003025.6, accessed 13 October 2020) and extracting the methylation ratio of every CpGs were conducted using BSMAP aligner (v2.87) (Baylor College of Medicine, Houston, TX, USA) [18].

To produce the transcriptome, RNA and cDNA library construction and RNA-seq were performed by TNT Research (Jeonju, Korea) as described [9,17], with total RNA isolated from the whole collected $\mathrm{CN}(n=3)$ and PA $(n=3)$ embryos using the TRIzol reagent (Sigma-Aldrich, St Louis, MO, USA) following the manufacturer's instructions. In brief, RNA samples treated with DNase I to avoid genomic DNA contamination were electrophoresed in $1.2 \%$ agarose gels to evaluate the integrity of RNA, which was then confirmed by the 28S/18S rRNA ratio (>2.0) and the RNA integrity number (RIN) (>7.0) using an Agilent 2100 BioAnalyzer. Using the ratios of A260/A280 and A260/A230 (1.8-2.0), the concentrations of RNA were assessed. One $\mu \mathrm{g}$ of total RNA was used to construct cDNA libraries with TruSeq RNA Sample Prep Kit v.2 (Illumina, San Diego, CA, USA). Quantification and qualification of the cDNA libraries were assessed by quantitative real-time PCR (qPCR) and using an Agilent 2100 Bioanalyzer, respectively. An Illumina HiSeq2500 RNA-seq platform was used to sequence the library products (101 nt pairedend). After quality checking, adapter trimming, and filtering by FastQC and Trim Galore, about 76.8 (CN1), 73.0 (CN2), 77.2 (CN3), 80.0 (PA1), 79.3 (PA2), and 80.3 (PA3) million cleaned reads were retrieved. STAR aligner (v.2.7.5) [19] was used to align the reads to the reference genome (Sscrofa11.1) with default parameter settings and produce BAM files of aligned reads. To normalize read coverages, deepTools (v3.5.0) [20] was used.

\subsection{Bioinformatics and Statistical Analysis}

All WGBS and RNA-seq alignments were visualized using the R/Bioconductor package Gviz (v1.32.0) [21] and/or Integrative Genomics Viewer (IGV) (v2.8.13) [22]. The downloaded raw RNA-seq and ChIP-seq data of adult pigs were analyzed to check tissuespecific and monoallelic expression. The downloaded reads were quality-checked by FastQC and trimmed using Trim Galore. STAR aligner [19] was used to map cleaned reads to the reference pig genome (Sscrofa11.1). Read coverages and alignments in those adult pigs were examined using BAM files and normalized using deepTools [20]. Differential expression was analyzed by quantifying the raw reads against the pig transcriptome using Salmon (v1.3.0) [23] and analyzing those quantified reads using the R/Bioconductor package DESeq2 (v.1.28.1) [24], and then the adjusted $p$-value $<0.05$ was regarded as statistically significant. To call a DMR with the threshold of $>10 \mathrm{CpGs}$, the maximum distance between CpGs $<300 \mathrm{bp}$, the mean methylation ratio difference $(\Delta$ ave $)>0.2$ and the false discovery rate $(\mathrm{FDR})<0.05$, metilene (v0.2-8) [25] was used. The shapiro.test function in the R package stats (v4.0.5) [26] was used to conduct the Shapiro-Wilk test to evaluate normality of the data. The Kruskal-Wallis test was performed using the kruskal.test function in the $\mathrm{R}$ package stats (v4.0.5) [26] to determine whether there is any significant difference between tissue groups regarding gene expression levels in adult pigs. 


\section{Results}

\subsection{Differential Expression and Methylation at the DIRAS3 Locus of Porcine Embryos}

To verify paternal expression of the DIRAS3 gene in day 21 porcine embryos, we compared DIRAS3 expression between our model of parthenogenetic embryos without the paternal allele and biparental control embryos. By analyzing RNA-seq data, an exclusive expression of DIRAS3 in the biparental control embryos with the paternal allele was found, but not in the parthenogenetic embryos (Figure 1a, Table 1). Surrounding noncoding genes (the porcine LOC110261211 and LOC110261446 genes encoding noncoding RNAs) were not expressed in both embryos. Furthermore, unlikely for humans, expression of an overlapping transcript (e.g., GNG12-AS1 in humans) was not detected. Further differential gene expression analysis revealed that the expression of DIRAS3 was more than 85-fold higher in the control embryos than in the parthenogenetic embryos (adjusted $p<0.05$ ) (Figure 1a). In addition, our analysis of WGBS showed that a significantly higher DNA methylation at the DIRAS3 locus occurred in the parthenogenetic embryos with two maternal alleles compared with the biparental control embryos (Figure 1b, Table 1, Table S1). This maternal hypermethylation established a DMR throughout the DIRAS3 locus including a CpG island. Taken together, our combined results of RNA-seq and WGBS provided strong evidence of maternal imprints that give rise to a paternal expression of the porcine DIRAS3 gene.

Table 1. DNA methylation and gene expression in PA and CN embryos.

\begin{tabular}{|c|c|c|c|c|c|c|}
\hline Gene/Locus & $\begin{array}{c}\text { Coordinate } \\
\text { (chr6) } \\
\text { Start \& End }\end{array}$ & Embryo & $\begin{array}{c}\text { Methylation } \\
\text { (Ratio, ave) }\end{array}$ & $\begin{array}{l}\text { Expression } \\
\text { (TPM, ave) }\end{array}$ & $p$-Value & $\begin{array}{c}\text { Adjusted } \\
p \text {-Value }\end{array}$ \\
\hline \multirow{2}{*}{ LOC110261211 } & \multirow{2}{*}{$\begin{array}{r}144,558,280 \\
144,571,021 \\
(12,741 \mathrm{bp})\end{array}$} & PA & $0.88 \pm 0.01$ & $0.16 \pm 0.00$ & \multirow{2}{*}{0.69} & \multirow{2}{*}{0.89} \\
\hline & & $\mathrm{CN}$ & $0.86 \pm 0.01$ & $0.18 \pm 0.05$ & & \\
\hline \multirow{2}{*}{ DMR } & \multirow{2}{*}{$\begin{array}{c}144,575,647 \\
144,578,475 \\
(2828 \mathrm{bp})\end{array}$} & PA & $0.95 \pm 0.00$ & NA & \multirow{2}{*}{ NA } & \multirow{2}{*}{ NA } \\
\hline & & $\mathrm{CN}$ & $0.50 \pm 0.01$ & NA & & \\
\hline \multirow{2}{*}{ DIRAS3 } & \multirow{2}{*}{$\begin{array}{c}144,576,342 \\
144,578,820 \\
(2478 \mathrm{bp})\end{array}$} & PA & $0.96 \pm 0.00$ & $1.00 \pm 0.17$ & \multirow{2}{*}{$<0.05$} & \multirow{2}{*}{$<0.05$} \\
\hline & & $\mathrm{CN}$ & $0.49 \pm 0.01$ & $85.78 \pm 1.55$ & & \\
\hline \multirow{3}{*}{ LOC110261446 } & \multirow{3}{*}{$\begin{array}{l}144,591,277 \\
144,721,840 \\
(130,563 \mathrm{bp})\end{array}$} & PA & $0.81 \pm 0.00$ & $0.00 \pm 0.00$ & \multirow{3}{*}{ NA } & \multirow{3}{*}{ NA } \\
\hline & & & & & & \\
\hline & & $\mathrm{CN}$ & $0.77 \pm 0.00$ & $0.00 \pm 0.00$ & & \\
\hline
\end{tabular}

PA, parthenogenetic embryo; $\mathrm{CN}$, control embryo. Values are presented as average (ave) \pm SEM. The $\mathrm{p}$-values and adjusted $\mathrm{p}$-values were obtained from DESeq2 for detecting differentially expressed genes. NA, not available. 


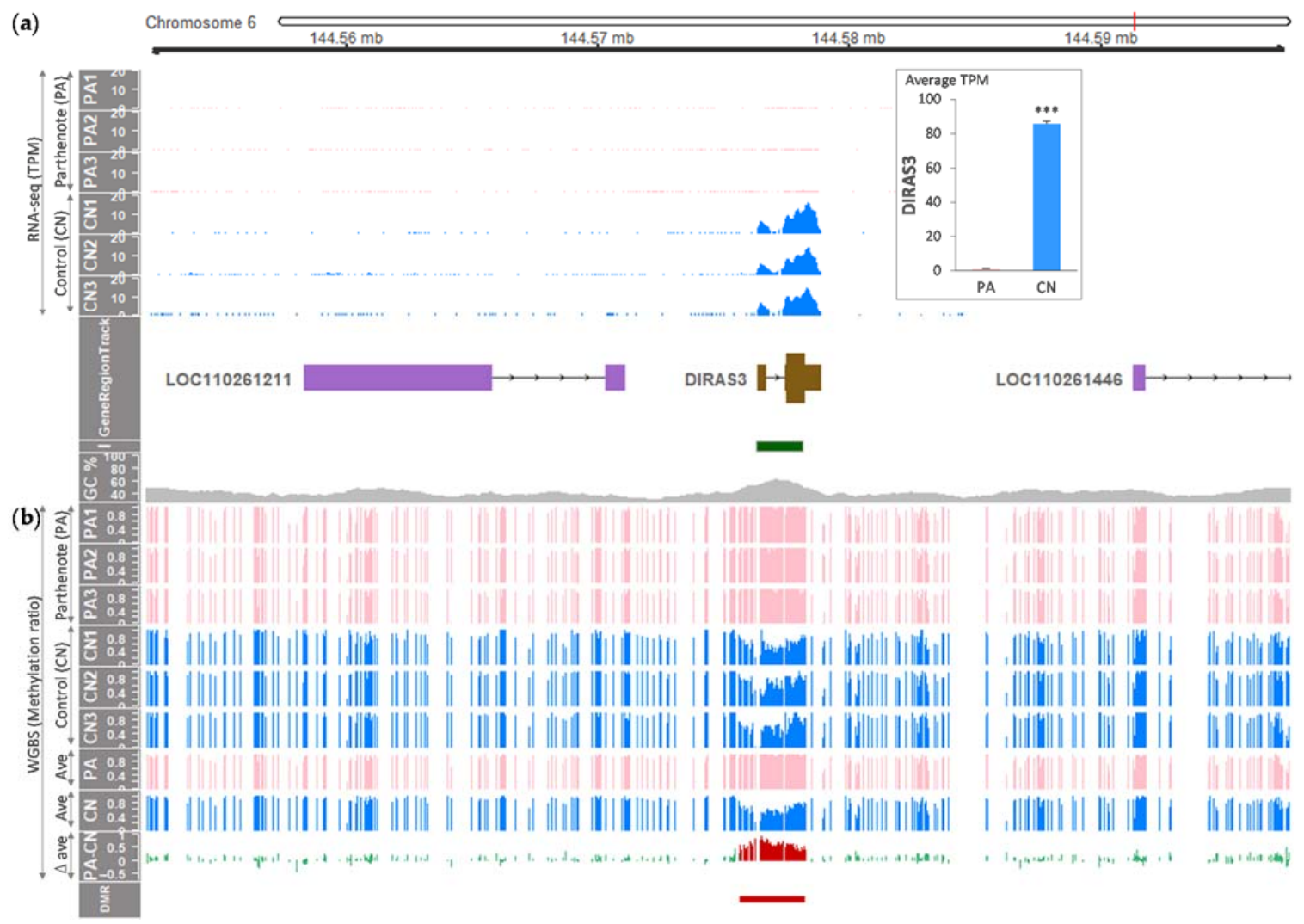

Figure 1. Profiling of the transcriptome and DNA methylome from the porcine parthenotes (PAs) and control embryos (CNs). (a) RNA-seq read coverages were normalized to transcripts per million (TPM). Differential expression (DE) of the porcine DIRAS3 gene between PA and CN $(n=3$ each) is shown in the box in the top-right corner (mean \pm SEM; $* * *$, adjusted $p<0.05$ ). In GeneRegionTrack, purple boxes indicate exons of noncoding genes, and a protein-coding gene is shown as brown boxes with tall and short boxes indicating translated and untranslated regions, respectively. The direction of transcription is marked by horizontal arrows. I, CpG islands; GC \%, GC content in percent. (b) CG methylation ratios of triplicates of PA and $\mathrm{CN}$ derived from whole-genome bisulfite sequencing (WGBS) are displayed, and averaged ratios were used to obtain differences in ratios ( $\Delta$ ave, delta average). A differentially methylated region (DMR) between PA and $\mathrm{CN}(\mathrm{FDR}<0.05)$ is indicated with a red bar and overlaid on differentially methylated CGs in the PA-CN track with red histogram lines.

\subsection{Sequence Elements and Differentially Methylated CpGs within the Porcine DIRAS3 Locus}

Since DNA hypermethylation has been recognized as an epigenetic regulator of gene expression that potentially silences or downregulates tumor suppressor genes such as DIRAS3 [27], detailed maternal hypermethylation status was profiled in the porcine DIRAS3 locus. Unlike humans in whom CpG islands are separated into three pieces at the promoters and the gene body, the DIRAS3 locus in pigs contains one consecutive CpG island spanning between a part of the promoter region and a large portion of the gene body (exon 1, intron, and exon 2) (Figure 2). Because differentially methylated CpGs (or $\mathrm{Cs}$ ) were distributed throughout the $\mathrm{CpG}$ island and even farther in the promoter and exon 2, a DMR was also consecutive throughout the porcine DIRAS3 locus. The consecutiveness was detected even with conservative criteria of at least ten differentially methylated CpGs with the maximum distance between CpGs of less than $300 \mathrm{bp}$, which was changed from the default maximum distance of $500 \mathrm{bp}$ in the DMR caller, metilene (Figure 2). On the other hand, in humans, three separate DMRs that corresponded to three CpG islands were reported [28]. In sum, in pig embryos, maternal hypermethylation occurred throughout the promoter and the gene body of the DIRAS3 gene beyond the CpG island, establishing one DMR. 


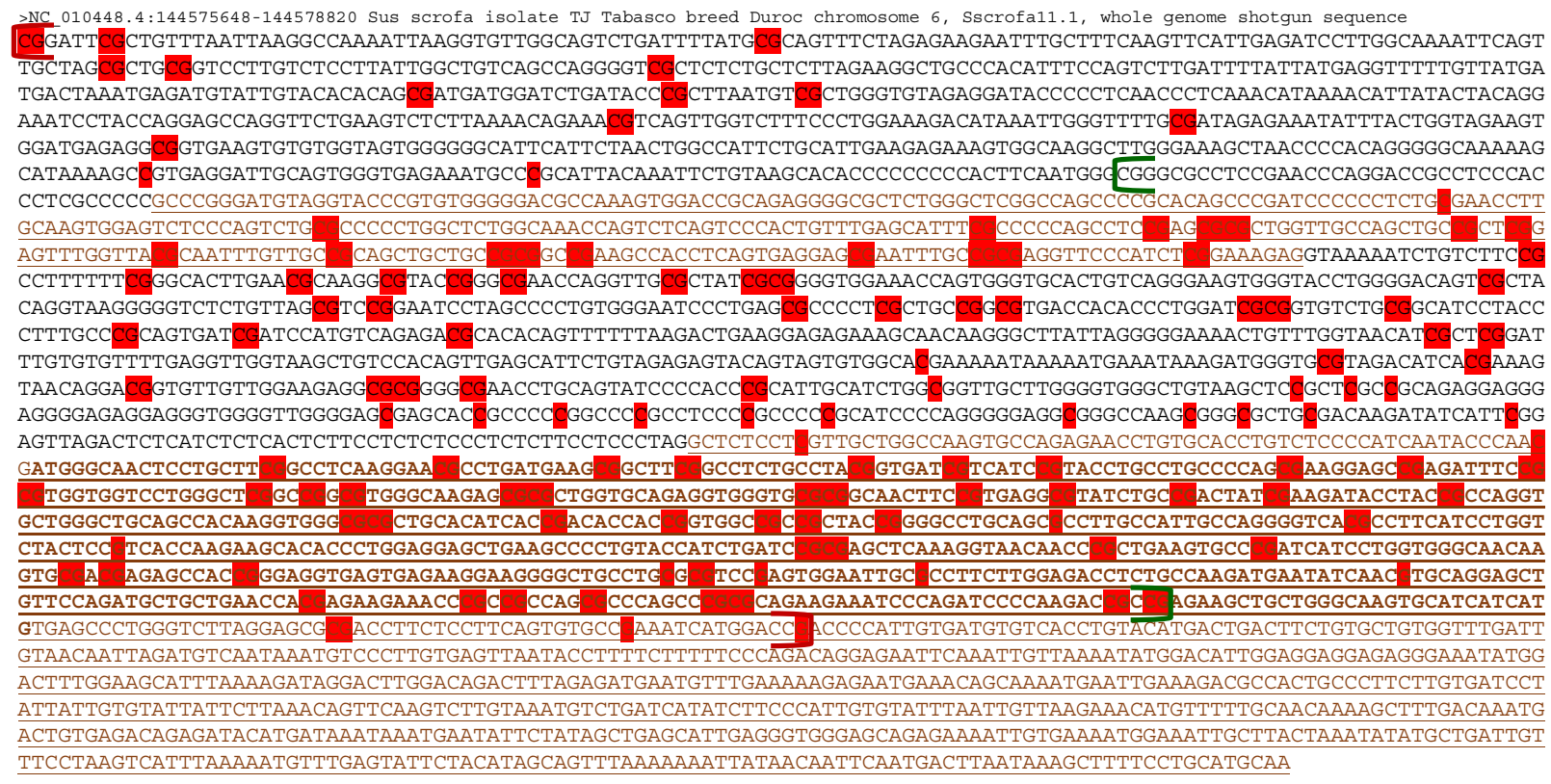

Figure 2. Distribution of differential methylation (i.e., maternal hypermethylation) in the DIRAS3 locus of the porcine parthenogenetic (PA) embryos compared to the control $(\mathrm{CN})$ embryos. The nucleotide sequence of the genomic DNA covers a promoter (black), exon 1 (brown, underlined), intron (black), and exon 2 (brown, underlined) of the porcine DIRAS3 gene from the beginning to the end. Brown bold faces in exon 2 indicate a translated region. This partial genomic DNA sequence in chromosome 6 (chr6 or NC_010448.4:144575648-144578820) of the reference pig genome (Sscrofa11.1 or susScr11) was retrieved from the NCBI Nucleotide database (https:/ / www.ncbi.nlm.nih.gov/nuccore, accessed 15 January 2021). The start and end of the CpG island (chr6:144576300-144578127) and DMR (displayed in Figure 1) are denoted with green and red brackets, respectively. Differentially methylated CpGs (or Cs) between PA and CN (i.e., hypermethylated in PA) are highlighted with red. Highlighted Gs indicate methylated Cs on the minus strand (coding strand). To call a DMR with a threshold, metilene was used, as mentioned in the Materials and Methods.

\subsection{Tissue-Specific and Monoallelic Expression of the DIRAS3 Gene in Adult Pigs}

We further examined the expression of DIRAS3 in adult pigs to show whether imprinted gene expression maintains at the adult stage. First, we explored whether tissuespecific expression patterns exist. Considering that the DIRAS3 gene in humans was expressed predominantly in the brain (hypothalamus, nucleus accumbens), as well as in the ovary and the pituitary (as mentioned above), we predicted that this expression pattern may be conserved in pigs. Our analysis of RNA-seq data from two six-month-old male pigs revealed the DIRAS3 gene expression appeared to be hypothalamus-specific among the analyzed major pig tissues (Table 2, Figure 3a,b). However, tissue distribution was non-normal according to the Shapiro-Wilk test, and therefore a non-parametric test (the Kruskal-Wallis test) was performed. The result showed that mRNA expressions of both LOC110261211 and DIRAS3 were not statistically different between the analyzed tissues (Table 2). On the other hand, these TPM values from the hypothalamus were approximately five to eight times higher than those values from the control embryos in Figure 1a. Among other tissues including adipose tissue, liver, lung, skeletal muscle, and spleen, DIRAS3 expression was observed as slightly higher in adipose tissue, but the absolute expression level was low (Figure 3a,b). Secondly, further analysis of read coverages and alignments showed that only reference alleles (A alleles in pig 1 and $C$ allele in pig 2) were expressed at heterozygous sites in all the tested tissues instead of the alternative alleles shown in published ChIP-seq data from genomic DNA (gDNA) of the same pigs, indicating monoallelic expression of the DIRAS3 gene (Figure 3c,d). Overall, these data suggest that the imprinted expression of DIRAS3 is maintained at the adult stage in which DIRAS3 expression shows a tendency of hypothalamus-enriched expression. 

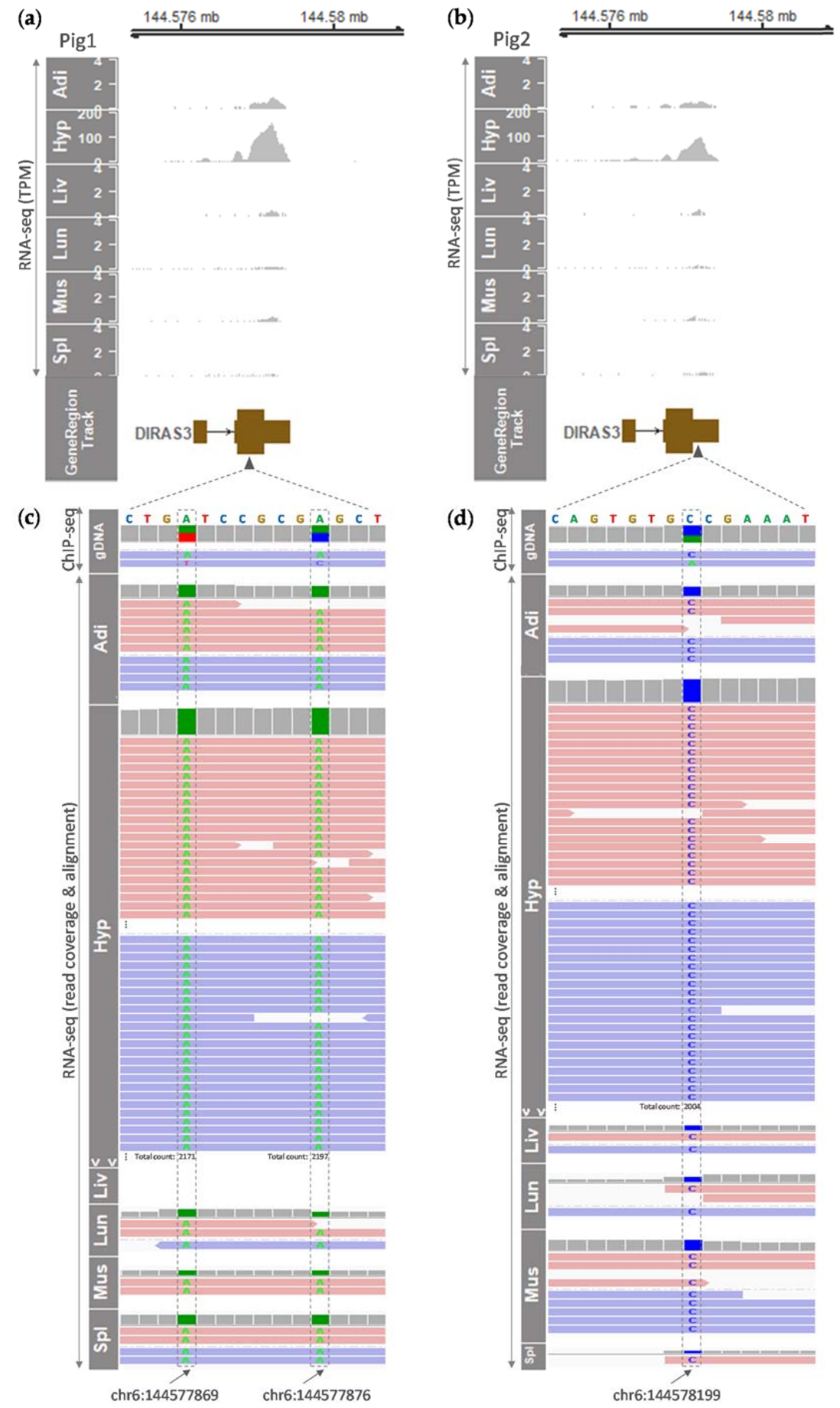

Figure 3. Tissue-specific and monoallelic expression of the DIRAS3 gene in adult pigs (six-month-old). Expressions of the DIRAS3 gene in various tissues from pig 1 (a) and pig 2 (b) were plotted based on TPM values obtained from RNA-seq. In addition, monoallelic expressions of the DIRAS3 gene in those various tissues were analyzed using read coverages and alignments in BAM files from RNA-seq as follows: for pig 1 (c), expression of reference alleles at two heterozygous sites (A on chr6:144577869; A on chr6:144577876) instead of alternative alleles ( $\mathrm{T}$ and C, respectively) is shown, and for pig 2 (d), expression of a reference allele at a heterozygous site (C on chr6:144578199) instead of an alternative allele (A) is displayed. Raw RNA-seq and ChIP-seq data were retrieved from GSE158430 and processed as described in the Materials and Methods. Adi, adipose tissue; gDNA, genomic DNA; Hyp, hypothalamus (brain); Liv, liver; Lun, lung; Mus, skeletal muscle; Spl, spleen. More reads were only present in the Hyp as indicated. Black arrowheads at the second exon of the DIRAS3 transcript denote locations of nucleotide sequences in $(\mathbf{c}, \mathbf{d})$. Black arrows at the bottom indicate genomic coordinates (chromosome and base position) of heterozygous sites. 
Table 2. Gene expression in various tissues of adult pigs.

\begin{tabular}{|c|c|c|c|c|c|c|}
\hline \multirow[b]{2}{*}{ Gene } & \multicolumn{6}{|c|}{ Expression (TPM, ave) } \\
\hline & $\begin{array}{l}\text { Adipose } \\
\text { Tissue }\end{array}$ & Hypothalamus & Liver & Lung & Muscle & Spleen \\
\hline LOC110261211 & $0.07 \pm 0.03$ & $0.17 \pm 0.09$ & $0.05 \pm 0.01$ & $0.12 \pm 0.01$ & $0.02 \pm 0.00$ & $0.11 \pm 0.02$ \\
\hline DIRAS3 & $1.60 \pm 0.30$ & $468.30 \pm 160.39$ & $0.26 \pm 0.04$ & $0.57 \pm 0.06$ & $0.34 \pm 0.09$ & $0.31 \pm 0.06$ \\
\hline LOC110261446 & $0.00 \pm 0.00$ & $0.00 \pm 0.00$ & $0.00 \pm 0.00$ & $0.00 \pm 0.00$ & $0.00 \pm 0.00$ & $0.00 \pm 0.00$ \\
\hline
\end{tabular}

For both LOC110261211 and DIRAS3, the distribution significantly differed from normal distribution according to the Shapiro-Wilk test ( $p$-values were 0.0423 and $1.267 \times 10^{-5}$, respectively). The $p$-values from the Kruskal-Wallis test were 0.148 (LOC110261211) and 0.08204 (DIRAS3), which are lower than the significance level (0.05) indicating that there were no significant differences between the tissue groups.

\section{Discussion}

Our findings in this study suggest that the DIRAS3 gene in porcine embryos is subjected to silencing of its promoter region in the maternal allele through DNA hypermethylation, which is one of the genomic imprinting mechanisms including direct silencing of promoters, inhibition of long-range communication between promoters and enhancers, repression of long-noncoding RNA (lncRNA), isoform-dependent silencing, and histone modifications [29-31]. This direct silencing via maternal DNA hypermethylation might lead to paternal allele-specific expression of DIRAS3 in biparental control embryos, but not in parthenogenetic embryos, where it is deficient in the paternal allele. As such, our generation of parthenogenetic embryos enabled detection of paternal expression by all-or-none fashion (i.e., all biparental controls having a paternal allele, and no parthenotes without any paternal allele), along with a construction of the methylome and the transcriptome.

Further analysis of DNA sequence elements and methylated CpGs showed that both the regions of $\mathrm{CpG}$ island and maternal methylation were more widely spread in pigs than in humans. Since aberrant DNA methylation on the paternal allele has been identified as a major inhibitor of the imprinted expression of DIRAS3 [14], a detailed overview of methylated regions is needed. It was reported that in human patients with breast cancer and ovarian cancer, hypermethylation in both CpG islands, I and II, was associated with reduced expression of DIRAS3 [32,33]. This CpG island II is located at the proximal promoter and exon 1 of the human DIRAS3 isoform [28,32] and corresponds, in part, to the pig C PG island, suggesting paternal hypermethylation in this region could be related to reduced DIRAS3 expression. Given that the porcine DMR was extended $652 \mathrm{bp}$ upstream and 86 bp downstream beyond the CpG island (Figure 2), there might be additional gene regulation via DNA methylation.

Furthermore, our data analysis revealed that in adult male pigs, the expression of the DIRAS3 gene tended to be hypothalamus-specific and monoallelic, suggesting maintenance of the imprinted gene expression during development. Despite the tendency of hypothalamus specificity, non-normal distribution and non-statistical significance $(p=0.08204)$ of the distribution of DIRAS3 might be due to the low sample size. Considering tissuespecific DIRAS3 expression patterns shown in the GTEx project (predominant in the human hypothalamus, nucleus accumbens, ovary, and pituitary) and given the hypothalamusspecific tendency in the current study, the reason why a previous study reported ubiquitous expression of porcine DIRAS3 in tested tissues [16] might be due to saturation of RT-PCR. There are also conflicting reports on the imprinted status of DIRAS3 in pig placenta, as one report mentioned a lack of imprinted isoform in the placenta [15], while another report displayed monoallelic expression of DIRAS3 in the placenta [34]. Based on our plot of RNA-seq read coverages, there was only one expressed transcript in the DIRAS3 locus, unlike humans in whom a longer isoform exists according to the Ensembl database (http:/ / www.ensembl.org/, accessed 15 January 2021). To clarify the imprinted status of one DIRAS3 transcript in the pig placenta, future studies will need to investigate both monoallelic expression and methylation status and link them to provide comprehensive profiling. 
Overall, the porcine DIRAS3 locus has similarities and differences compared to the human ortholog. The presence of maternal hypermethylation (imprints), tissue-specific expression tendency in the hypothalamus, and its monoallelic expression pattern were similar. Differences covered the locations and numbers of the CpG island and DMR, as well as non-existence of an overlapping GNG12-AS transcript in pigs. Further investigation across species in a comparative manner will improve our understanding on the underlying genetic and epigenetic mechanisms related to the DIRAS3 locus.

\section{Conclusions}

Although genes imprinted in humans and mice have been widely characterized, including tumor suppressor genes such as DIRAS3 or ARHI, there is a lack of detailed and comprehensive studies in livestock species including pigs. The findings from this study suggest that the presence of maternal hypermethylation, or imprints, might be maintained and related to monoallelic expression of DIRAS3 during pig development. Our approach based on parthenogenesis and various next-generation sequencing data promotes comprehensive and detailed identification of imprinted genes and loci in a genomic context.

Supplementary Materials: The following are available online at https:/ / www.mdpi.com/article/10.3 390/ani11051315/s1, Table S1: Differentially methylated region (DMR) called by the metilene software.

Author Contributions: Conceptualization, K.L.; methodology, J.A.; software, J.A.; validation, K.L. and J.A.; formal analysis, J.A.; investigation, J.A.; resources, I.-S.H. and M.-R.P.; S.H., and K.L.; data curation, J.A.; writing-original draft preparation, J.A.; writing —review and editing, K.L. and J.A.; visualization, J.A.; supervision, K.L.; project administration, K.L.; funding acquisition, K.L. All authors have read and agreed to the published version of the manuscript.

Funding: This research was funded by the United States Department of Agriculture's National Institute of Food and Agriculture's Hatch Grant (project No. OHO01304).

Institutional Review Board Statement: The study was conducted according to the guidelines that were approved by the Institutional Animal Care and Use Committee of the National Institute of Animal Science, Rural Development Administration (RDA) of Korea (approval number NIAS2015-670).

Data Availability Statement: Data supporting the results were downloaded from GSE158430 and can be found within the Supplementary Materials.

Acknowledgments: We are grateful to Michelle Milligan for her invaluable assistance of proofreading this manuscript.

Conflicts of Interest: The authors declare no conflict of interest.

\section{References}

1. Franklin, G.C.; Adam, G.I.; Ohlsson, R. Genomic imprinting and mammalian development. Placenta 1996, 17, 3-14. [CrossRef]

2. Greenberg, M.V.C.; Bourc'his, D. The diverse roles of DNA methylation in mammalian development and disease. Nat. Rev. Mol. Cell Biol 2019, 20, 590-607. [CrossRef]

3. Zeng, Y.; Chen, T. DNA Methylation Reprogramming during Mammalian Development. Genes 2019, 10, 257. [CrossRef] [PubMed]

4. da Rocha, S.T.; Gendrel, A.V. The influence of DNA methylation on monoallelic expression. Essays Biochem. 2019, 63, 663-676. [CrossRef] [PubMed]

5. Ono, R.; Kobayashi, S.; Wagatsuma, H.; Aisaka, K.; Kohda, T.; Kaneko-Ishino, T.; Ishino, F. A retrotransposon-derived gene, PEG10, is a novel imprinted gene located on human chromosome 7q21. Genomics 2001, 73, 232-237. [CrossRef] [PubMed]

6. Grabowski, M.; Zimprich, A.; Lorenz-Depiereux, B.; Kalscheuer, V.; Asmus, F.; Gasser, T.; Meitinger, T.; Strom, T.M. The epsilonsarcoglycan gene (SGCE), mutated in myoclonus-dystonia syndrome, is maternally imprinted. Eur. J. Hum. Genet. 2003, 11, 138-144. [CrossRef] [PubMed]

7. Piras, G.; El Kharroubi, A.; Kozlov, S.; Escalante-Alcalde, D.; Hernandez, L.; Copeland, N.G.; Gilbert, D.J.; Jenkins, N.A.; Stewart, C.L. Zac1 (Lot1), a potential tumor suppressor gene, and the gene for epsilon-sarcoglycan are maternally imprinted genes: Identification by a subtractive screen of novel uniparental fibroblast lines. Mol. Cell. Biol. 2000, 20, 3308-3315. [CrossRef]

8. Ono, R.; Shiura, H.; Aburatani, H.; Kohda, T.; Kaneko-Ishino, T.; Ishino, F. Identification of a large novel imprinted gene cluster on mouse proximal chromosome 6. Genome Res. 2003, 13, 1696-1705. [CrossRef]

9. Ahn, J.; Hwang, I.S.; Park, M.R.; Cho, I.C.; Hwang, S.; Lee, K. The Landscape of Genomic Imprinting at the Porcine SGCE/PEG10 Locus from Methylome and Transcriptome of Parthenogenetic Embryos. G3 (Bethesda) 2020. [CrossRef] 
10. Duan, J.E.; Zhang, M.; Flock, K.; Seesi, S.A.; Mandoiu, I.; Jones, A.; Johnson, E.; Pillai, S.; Hoffman, M.; McFadden, K.; et al. Effects of maternal nutrition on the expression of genomic imprinted genes in ovine fetuses. Epigenetics 2018, 13, 793-807. [CrossRef] [PubMed]

11. Li, X.; Liu, S.; Fang, X.; He, C.; Hu, X. The mechanisms of DIRAS family members in role of tumor suppressor. J. Cell. Physiol. 2019, 234, 5564-5577. [CrossRef] [PubMed]

12. Lu, Z.; Bast, R.C., Jr. The tumor suppressor gene ARHI (DIRAS3) inhibits ovarian cancer cell migration through multiple mechanisms. Cell Adh. Migr. 2013, 7, 232-236. [CrossRef] [PubMed]

13. Yu, Y.; Xu, F.; Peng, H.; Fang, X.; Zhao, S.; Li, Y.; Cuevas, B.; Kuo, W.L.; Gray, J.W.; Siciliano, M.; et al. NOEY2 (ARHI), an imprinted putative tumor suppressor gene in ovarian and breast carcinomas. Proc. Natl. Acad. Sci. USA 1999, 96, 214-219. [CrossRef]

14. Xu, F.; Xia, W.; Luo, R.Z.; Peng, H.; Zhao, S.; Dai, J.; Long, Y.; Zou, L.; Le, W.; Liu, J.; et al. The human ARHI tumor suppressor gene inhibits lactation and growth in transgenic mice. Cancer Res. 2000, 60, 4913-4920.

15. Bischoff, S.R.; Tsai, S.; Hardison, N.; Motsinger-Reif, A.A.; Freking, B.A.; Nonneman, D.; Rohrer, G.; Piedrahita, J.A. Characterization of conserved and nonconserved imprinted genes in swine. Biol. Reprod. 2009, 81, 906-920. [CrossRef]

16. Cheng, H.C.; Zhang, F.W.; Deng, C.Y.; Jiang, C.D.; Xiong, Y.Z.; Li, F.E.; Lei, M.G. NNAT and DIRAS3 genes are paternally expressed in pigs. Genet. Sel. Evol. 2007, 39, 599-607. [CrossRef]

17. Ahn, J.; Wu, H.; Lee, J.; Hwang, I.S.; Yu, D.; Ahn, J.S.; Lee, J.W.; Hwang, S.; Lee, K. Identification of a Novel Imprinted Transcript in the Porcine GNAS Complex Locus Using Methylome and Transcriptome of Parthenogenetic Fetuses. Genes 2020, 11, 96. [CrossRef]

18. Xi, Y.; Li, W. BSMAP: Whole genome bisulfite sequence MAPping program. BMC Bioinform. 2009, 10, 232. [CrossRef]

19. Dobin, A.; Davis, C.A.; Schlesinger, F.; Drenkow, J.; Zaleski, C.; Jha, S.; Batut, P.; Chaisson, M.; Gingeras, T.R. STAR: Ultrafast universal RNA-seq aligner. Bioinformatics 2013, 29, 15-21. [CrossRef] [PubMed]

20. Ramirez, F.; Dundar, F.; Diehl, S.; Gruning, B.A.; Manke, T. deepTools: A flexible platform for exploring deep-sequencing data. Nucleic Acids Res. 2014, 42, W187-W191. [CrossRef]

21. Hahne, F.; Ivanek, R. Visualizing Genomic Data Using Gviz and Bioconductor. Methods Mol. Biol. 2016, 1418, 335-351. [CrossRef] [PubMed]

22. Thorvaldsdottir, H.; Robinson, J.T.; Mesirov, J.P. Integrative Genomics Viewer (IGV): High-performance genomics data visualization and exploration. Brief. Bioinform. 2013, 14, 178-192. [CrossRef]

23. Patro, R.; Duggal, G.; Love, M.I.; Irizarry, R.A.; Kingsford, C. Salmon provides fast and bias-aware quantification of transcript expression. Nat. Methods 2017, 14, 417-419. [CrossRef] [PubMed]

24. Love, M.I.; Huber, W.; Anders, S. Moderated estimation of fold change and dispersion for RNA-seq data with DESeq2. Genome Biol. 2014, 15, 550. [CrossRef] [PubMed]

25. Juhling, F.; Kretzmer, H.; Bernhart, S.H.; Otto, C.; Stadler, P.F.; Hoffmann, S. metilene: Fast and sensitive calling of differentially methylated regions from bisulfite sequencing data. Genome Res. 2016, 26, 256-262. [CrossRef]

26. RCoreTeam. R: A language and environment for statistical computing. R Foundation for Statistical Computing. Vienna, Austria, 2021. Available online: https:/ /www.R-project.org/ (accessed on 29 April 2021).

27. Klett, H.; Balavarca, Y.; Toth, R.; Gigic, B.; Habermann, N.; Scherer, D.; Schrotz-King, P.; Ulrich, A.; Schirmacher, P.; Herpel, E.; et al. Robust prediction of gene regulation in colorectal cancer tissues from DNA methylation profiles. Epigenetics 2018, 13, 386-397. [CrossRef]

28. Niemczyk, M.; Ito, Y.; Huddleston, J.; Git, A.; Abu-Amero, S.; Caldas, C.; Moore, G.E.; Stojic, L.; Murrell, A. Imprinted chromatin around DIRAS3 regulates alternative splicing of GNG12-AS1, a long noncoding RNA. Am. J. Hum. Genet. 2013, 93, 224-235. [CrossRef]

29. Barlow, D.P.; Bartolomei, M.S. Genomic imprinting in mammals. Cold Spring Harb. Perspect. Biol. 2014, 6. [CrossRef]

30. Tian, X.C. Genomic imprinting in farm animals. Annu. Rev. Anim. Biosci. 2014, 2, 23-40. [CrossRef]

31. Tucci, V.; Isles, A.R.; Kelsey, G.; Ferguson-Smith, A.C.; Erice Imprinting, G. Genomic Imprinting and Physiological Processes in Mammals. Cell 2019, 176, 952-965. [CrossRef]

32. Feng, W.; Marquez, R.T.; Lu, Z.; Liu, J.; Lu, K.H.; Issa, J.P.; Fishman, D.M.; Yu, Y.; Bast, R.C., Jr. Imprinted tumor suppressor genes ARHI and PEG3 are the most frequently down-regulated in human ovarian cancers by loss of heterozygosity and promoter methylation. Cancer 2008, 112, 1489-1502. [CrossRef] [PubMed]

33. Yuan, J.; Luo, R.Z.; Fujii, S.; Wang, L.; Hu, W.; Andreeff, M.; Pan, Y.; Kadota, M.; Oshimura, M.; Sahin, A.A.; et al. Aberrant methylation and silencing of ARHI, an imprinted tumor suppressor gene in which the function is lost in breast cancers. Cancer Res. 2003, 63, 4174-4180. [PubMed]

34. Zhou, Q.Y.; Fang, M.D.; Huang, T.H.; Li, C.C.; Yu, M.; Zhao, S.H. Detection of differentially expressed genes between Erhualian and Large White placentas on day 75 and 90 of gestation. BMC Genom. 2009, 10, 337. [CrossRef] [PubMed] 